a retrospective review of a prospectively maintained database of patients who underwent resection of CLM during the period 19851994. The authors identified 102 actual 10year survivors, 99 of whom were disease free at last follow-up. Only one patient experienced a disease-specific death after 10 years of survival. By contrast, 34\% of 5-year survivors suffered a cancer-related death beyond 5 years. The presence of poor prognostic factors in the 10-year survivors, such as synchronous disease, bilobar metastases, node-positive primary tumor, more than one metastases, and hepatic tumor size larger than $5 \mathrm{~cm}$ did not preclude the possibility of long-term survival and cure.

The authors conclude that a third of patients who survive 5 years succumb to a cancerrelated death, but those who survive 10 years seem to be cured. Patients carefully selected for CLM resection have at least a $17 \%$ chance of cure.

Original article Tomlinson JS et al. (2007) Actual 10-year survival after resection of colorectal liver metastases defines cure. J Clin Oncol 25: 4575-4580

\section{Level of HER2 amplification correlates with response to trastuzumab-based therapy}

In patients with HER2-positive breast cancer, trastuzumab-based therapies offer substantial clinical benefits; however, little is known about the relationship between HER2 levels and pathological response. Arnould et al. recently analyzed the association between HER2 amplification levels and pathologic complete response in locally advanced breast cancer.

Using fluorescence in situ hybridization (FISH) and immunohistochemistry, HER2 amplification and overexpression were analyzed in biopsies obtained from 93 patients who had received trastuzumab in combination with chemotherapy for HER2-positive stage II/III breast cancer. Overall, 50 samples showed high HER2 amplification, while 27 and 16 samples were classified as low and no amplification, respectively. There was a significant correlation between the presence of HER2 amplification and pathologic complete response $(P<0.001)$. Tumors with $H E R 2$ amplification had significantly higher pathologic complete response than did tumors with no HER2 amplification on FISH analysis (44\% and $6 \%$, respectively; $P=0.004$ ). The level of HER2 amplification (high vs low) as assessed by FISH was the only variable related to complete response $(P=0.005)$; treatment regimen, patient age, $\mathrm{T}$ or $\mathrm{N}$ stage, hormone receptor status, tumor grade and immunohistochemical score were not significantly correlated with pathologic complete response.

This is the first study to show that levels of HER2 amplification, as assessed by FISH, have a positive correlation with the rate of pathologic complete response in patients treated with trastuzumab-based neoadjuvant therapy for stage II/III breast cancer. These findings might have important clinical implications in the treatment of patients with locally advanced HER2-positive disease.

Original article Arnould L et al. (2007) Pathologic complete response to trastuzumab-based neoadjuvant therapy is related to the level of HER2 amplification. Clin Cancer Res 13: 6404-6409

\section{Study identifies 10-gene signature prognostic for survival in stage I lung adenocarcinoma}

The benefit of adjuvant treatment in lung adenocarcinoma is currently unclear, as clinical trials have produced conflicting results. Reliable prognostic indicators might increase the power of clinical trials to prove the benefit of multimodality therapy by enabling the selection of only those patients at high risk of relapse following surgery. Bianchi et al. report a 10-gene prognostic signature for stage I lung adenocarcinoma.

To determine the signature, the researchers first conducted meta-analyses on two published gene-expression datasets from lung adenocarcinomas. These analyses identified 49 genes that were differentially expressed in patients with good versus poor prognoses. In the second stage of the strategy, the 49-gene model was pooled with 28 genes previously identified from a model that mimics cancerrelated pathways and 3 genes identified in the literature as individual prognostic markers.

To enable easy use of the signature in the clinical laboratory, the resulting 80 -gene model was refined to a 10-gene model through the leave-one-out validation method in a cohort of patients with stage I adenocarcinoma. In 